\title{
One Hundred
}

National Cancer Institute

\section{Source}

National Cancer Institute. One Hundred. NCI Thesaurus. Code C105814.

A natural number greater than ninety-nine and less than one hundred-one and the quantity that it denotes. 